\title{
Variability of longitudinal strain in left ventricular segments supplied by non-stenosed coronary artery: insights from speckle tracking analysis of dobutamine stress echocardiograms in patients with high coronary risk profile
}

\author{
Karina Wierzbowska-Drabik, Michał Plewka, Jarosław D. Kasprzak
}

\section{Chair and Department of Cardiology, Medical University of Lodz, Lodz, Poland}

Submitted: 12 January 2015

Accepted: 14 February 2015

Arch Med Sci 2017; 13, 1: 82-92

DOI: 10.5114 /aoms.2016.60603

Copyright (c) 2016 Termedia \& Banach

\section{Abstract}

Introduction: Although global deformation parameters have been increasingly used for myocardial function analysis, there are sparse data concerning segmental deformation of the left ventricle (LV). Moreover, some studies suggest heterogeneity of strain among LV segments, which may be especially significant during stress echocardiography. We assessed quantitatively regional LV function in the setting of dobutamine stress echocardiography (DSE), to examine differences of longitudinal strain between basal, mid and apical LV segments and to compare variability of regional deformation between rest and the peak stage of DSE.

Material and methods: Among 250 patients examined by DSE applied for diagnosis of ischemia, a subset of 111 patients without significant coronary stenoses in angiography was selected (68 females, mean age: $60 \pm 10$ years). Systolic longitudinal strain (SLS) in individual LV segments at baseline and the peak stage of DSE was analyzed with speckle tracking echocardiography. Results: Inhomogeneity of SLS among the LV segments $(p<0.001)$ was observed at baseline and the peak stage. Dispersion indices were higher at the peak stage of DSE than at baseline $(p<0.001)$, and the lowest heterogeneity was observed among mid segments. The analysis of changes in SLS during DSE showed SLS reduction in basal and mid-ventricular segments and an increase in apical segments.

Conclusions: Significant heterogeneity of strain and the opposite direction of the longitudinal strain changes during DSE between apical and basal LV segments were observed. This variability among non-ischemic LV segments ought to be considered in quantification of LV function during DSE.

Key words: strain, dobutamine stress echocardiography, segmental variability of strain.

\section{Introduction}

Despite the development of echocardiographic quantitative methods for myocardial function assessment, the subjective, visual analysis of thickening remains the standard for the evaluation of left ventricular (LV) contractility during stress testing [1-10]. Previous studies revealed inhomogeneity of regional myocardial velocities defined as criteria for im-

\section{Corresponding author:}

Karina Wierzbowska-Drabik MD

Chair and Department

of Cardiology

Medical University of Lodz

$1 / 5$ Kniaziewicza St

Lodz, Poland

Phone: 484221398 91,

504667844

E-mail: wierzbowska@

ptkardio.pl 
paired function during stress. Katz et al. proposed velocities in basal and middle LV segments below $5.5 \mathrm{~cm} / \mathrm{s}$ as the criterion of ischemia during dobutamine stress echocardiography (DSE) [11]. Cain et al. defined a cut-off $<7 \mathrm{~cm} / \mathrm{s}$ in basal septal and inferior walls, $<5 \mathrm{~cm} / \mathrm{s}$ in mid segments of these walls, $<6 \mathrm{~cm} / \mathrm{s}$ for basal segments of anterior, lateral and posterior walls, and $<4 \mathrm{~cm} / \mathrm{s}$ in mid anterior, lateral and posterior segments [12]. However, tissue Doppler parameters provided lower than expected sensitivity when tested in the MYDISE study [13]. The introduction of deformation parameters offered the advantage of independence from tethering by adjacent segments. When calculated as a conversion of tissue Doppler velocities, they are still dependent on the angle of insonation and less useful for the apical region [14-19]. Recently, a method based on two-dimensional speckle tracking echocardiography (STE) has been introduced with modifications, including automated function imaging (AFI), delivering results in the ser-friendly form of polar maps [20-23]. Nevertheless, whereas the assessment of global LV function has started to be incorporated into clinical practice, there is still a paucity of data concerning more time-consuming and hard to interpret regional deformation. Especially the data concerning dynamic changes of segmental strain during stress echocardiography are very limited [24]. The changes of regional deformation between baseline and peak stages of a stress test have not been described, and the potential differences for various stressors (exercise, dobutamine, rapid pacing, dipyridamole) are awaiting examination. Published data suggest some segmental heterogeneity of deformation parameters, which may impede the identification of universal cut-off values for the detection of ischemia.

That was why the aim of our study was to analyze regional values and the changes of systolic longitudinal strain (SLS) of each particular segment of the LV at rest and at the peak stage of DSE and to test the hypothesis of their homogeneity in the setting of a stress test.

\section{Material and methods}

\section{Study group and protocol}

We performed DSE with early atropine administration in 250 consecutive subjects with symptoms of angina. The protocol included: physical examination, electrocardiography and basic laboratory data, transthoracic echocardiography and DSE. All patients were in a sinus rhythm and free of significant valve disease. Further analysis included 238 patients with diagnostic stress echocardiography - when the test was terminated because of ischemia or reaching the age-predicted heart rate limit $(85 \% \times(220-$ age $))$. All these patients had an angiographic examination of coronary arteries no later than three months after DSE. A diameter stenosis of $\geq 50 \%$ in the left main coronary artery or $\geq 70 \%$ in other epicardial arteries was defined as significant. After a coronary imaging study, we selected 111 patients (68 female, mean age: 60 \pm 10 years) without significant lesions in coronary arteries to analyze the regional longitudinal strain at rest and the peak stage of the stress test, as well as the changes of deformation parameters between baseline and the peak level of DSE. The study was limited to 111 subjects without significant coronary artery stenoses to avoid the influence of ischemia on regional LV function. We calculated the indices of dispersion for comparison of segmental deformation heterogeneity at rest and stress and for basal, mid and apical regions of the LV. The demographic characteristics of the group without coronary stenoses are presented in Table I and the echocardiographic parameters in Table II. All subjects gave written informed consent to participate in the study, and the protocol was approved by the Ethical Commission of the Medical University of Lodz (number of agreement RNN/119/10 KE from 13.07.2010).

\section{Echocardiographic assessment}

We used an 18-segment model of LV, dividing each wall into three segments: basal, mid and apical. The evaluation of systolic function was performed by consensual visual assessment of two expert readers.

\section{Dobutamine stress echocardiography}

Dobutamine was administered by intravenous infusion in doses of $10,20,30$ and $40 \mu \mathrm{g} / \mathrm{kg} / \mathrm{min}$ during 3-minute stages, and atropine was added in $0.5 \mathrm{mg}$ boluses after the second stage up to the dose of $2 \mathrm{mg}$. Blood pressure was measured during each stage, and 12-lead ECG was recorded immediately after termination of the test.

The infusion was stopped when induced wall motion abnormalities suggestive for ischemia, chest pain, arrhythmia, excessive blood pressure increase or hypotension occurred or the age-predicted heart rate was reached. Video loops of heart cycles were acquired using standard echocardiographic views (three apical and LV short-axis view at three levels) and digitally stored for further analysis.

\section{Assessment of myocardial deformation}

Calculation of deformation parameters was performed off-line on an EchoPac 6.1.0 workstation (GE Vingmed Ultrasound). SLS values were obtained by 2D STE requiring manual tracing of en- 
Table I. Demographic characteristics, clinical data and treatment of the studied subjects without significant coronary stenoses

\begin{tabular}{|c|c|c|}
\hline $\begin{array}{l}\text { Parameter in studied } \\
\text { group }(n=111)\end{array}$ & Mean \pm SD & Range \\
\hline Age [years] & $60 \pm 10$ & $38-79$ \\
\hline Height $[\mathrm{cm}]$ & $167 \pm 8$ & $150-187$ \\
\hline Body mass [kg] & $79 \pm 15$ & $45-115$ \\
\hline Body mass index $\left[\mathrm{kg} / \mathrm{m}^{2}\right]$ & $29 \pm 5$ & $18-44$ \\
\hline Body surface area $\left[\mathrm{m}^{2}\right]$ & $1.92 \pm 0.21$ & $1.41-2.43$ \\
\hline $\begin{array}{l}\text { Blood pressure systolic } \\
{[\mathrm{mm} \mathrm{Hg}]}\end{array}$ & $127 \pm 17$ & $90-170$ \\
\hline $\begin{array}{l}\text { Blood pressure diastolic } \\
{[\mathrm{mm} \mathrm{Hg}]}\end{array}$ & $71 \pm 10$ & $43-100$ \\
\hline Heart rate $\left[\mathrm{min}^{-1}\right]$ & $66 \pm 10$ & $40-86$ \\
\hline Total cholesterol [mg/dl] & $207 \pm 42$ & $96-331$ \\
\hline Cholesterol LDL [mg/dl] & $122 \pm 33$ & $40-212$ \\
\hline Cholesterol HDL [mg/dl] & $56 \pm 12$ & $31-95$ \\
\hline Parameter & $N$ or $n(\%)$ & Percentage \\
\hline Female/male & $68 / 43$ & $61 / 39$ \\
\hline \multicolumn{3}{|l|}{ Chest pain: } \\
\hline Typical & 36 & 32 \\
\hline Atypical & 58 & 52 \\
\hline Non-specific & 17 & 15 \\
\hline Hypertension & 87 & 78 \\
\hline Diabetes & 25 & 23 \\
\hline Smoking & 53 & 48 \\
\hline Hypercholesterolemia & 88 & 79 \\
\hline $\begin{array}{l}\text { Family history of coronary } \\
\text { disease }\end{array}$ & 20 & 18 \\
\hline Acetylsalicylic acid & 83 & 75 \\
\hline Clopidogrel & 10 & 9 \\
\hline$\beta$-Adrenolytic & 63 & 57 \\
\hline ACE inhibitor & 68 & 61 \\
\hline Statin & 80 & 72 \\
\hline Long-acting nitrates & 22 & 20 \\
\hline
\end{tabular}

docardium in three apical views. In order to make the process of obtaining regional data more efficient, we assigned LV segments in clockwise order starting with the basal septum in the 4-chamber view, through the 2-chamber to 3-chamber view with consecutive numbers - see polar maps in Figures $1-3$. For each LV segment the peak SLS values were measured at baseline and the peak level of DSE (SLS $S_{B}, S L S_{P}$ respectively) as maximal values recorded before aortic valve closure.

The alternative method for obtaining SLS, i.e. $A F I$, required indicating three points (two basal
Table II. Echocardiographic parameters of the stud ied subjects without significant coronary stenoses

\begin{tabular}{|c|c|c|}
\hline $\begin{array}{l}\text { Parameter in studied } \\
\text { group }(n=111)\end{array}$ & Mean \pm SD & Range \\
\hline $\mathrm{LVd}[\mathrm{mm}]$ & $46.5 \pm 4.5$ & $35-58$ \\
\hline LVs [mm] & $31.7 \pm 4.7$ & $22-47$ \\
\hline $\mathrm{PWd}[\mathrm{mm}]$ & $10.9 \pm 1.5$ & $8-15$ \\
\hline PWs $[\mathrm{mm}]$ & $14.0 \pm 1.6$ & $10-19$ \\
\hline IVSd [mm] & $11.3 \pm 1.7$ & $8-15$ \\
\hline IVSs [mm] & $14.3 \pm 1.6$ & $11-19$ \\
\hline Ao $[\mathrm{mm}]$ & $32.1 \pm 3.8$ & $25-45$ \\
\hline $\mathrm{LA}[\mathrm{mm}]$ & $39.4 \pm 3.9$ & $30-51$ \\
\hline $\mathrm{RV}[\mathrm{mm}]$ & $26.0 \pm 2.2$ & $20-30$ \\
\hline$E / A$ & $0.94 \pm 0.3$ & $0.4-1.9$ \\
\hline LV mass $[g]$ & $223 \pm 62$ & $102-392$ \\
\hline LV mass index $\left[\mathrm{g} / \mathrm{m}^{2}\right]$ & $116.2 \pm 27.9$ & 59-195 \\
\hline ESV at baseline [ml] & $22.1 \pm 9.2$ & $8-62$ \\
\hline EDV at baseline [ml] & $54.3 \pm 15.8$ & $28-105$ \\
\hline SV at baseline [ml] & $32.4 \pm 9.6$ & $14-61$ \\
\hline EF at baseline (\%) & $60 \pm 9.0$ & $37-76$ \\
\hline WMSI at baseline & $1.04 \pm 0.09$ & $1.0-1.5$ \\
\hline $\mathrm{S}^{\prime}$ lat at baseline $[\mathrm{cm} / \mathrm{s}]$ & $8.7 \pm 2.2$ & $5-14$ \\
\hline E' lat at baseline $[\mathrm{cm} / \mathrm{s}]$ & $10.6 \pm 2.7$ & $4-18$ \\
\hline ESV at peak [ml] & $12.9 \pm 5.5$ & $4-31$ \\
\hline EDV at peak [ml] & $39.5 \pm 12.6$ & $11-74$ \\
\hline SV at peak [ml] & $26.8 \pm 9.2$ & $6-47$ \\
\hline EF at peak (\%) & $67.8 \pm 8.2$ & $44-85$ \\
\hline WMSI at peak & $1.08 \pm 0.11$ & $1.0-1.5$ \\
\hline $\mathrm{S}^{\prime}$ lat at peak $[\mathrm{cm} / \mathrm{s}]$ & $14.9 \pm 4.1$ & $5-23$ \\
\hline E' lat at peak [cm/s] & $15.5 \pm 3.6$ & $7-22$ \\
\hline
\end{tabular}

$N$ - number of subjects, LVd - left ventricular end diastolic dimension, LVs - left ventricular end systolic dimension, PWd - end diastolic left ventricular posterior wall thickness, PWs - end systolic left ventricular posterior wall thickness, IVSd - end diastolic left ventricular septum thickness, IVSs - end systolic left ventricular septum thickness, Ao - aortic dimension, LA - left atrial dimension, $R V$ - right ventricular end diastolic dimension, E/A - ratio of early to atrial mitral inflow peak velocity, LV mass - left ventricular mass, $L V$ mass index - left ventricular mass index, ESV - end systolic left ventricular volume, EDV - end diastolic left ventricular volume, $S V$ - stroke volume, EF - left ventricular ejection fraction, WMSI - wall motion score index, S' lat - peak systolic velocity of lateral part of mitral annulus, E' lat - peak early diastolic velocity of lateral part of mitral annulus.

and one apical) in each apical view and accepting the proposed region of interest encompassing the myocardial width. The regional values of peak SLS were presented in the format of a polar LV map with information describing averaged (from 6 segments) and global (from 18 segments) parameters. 
Variability of longitudinal strain in left ventricular segments supplied by non-stenosed coronary artery: insights from speckle tracking analysis of dobutamine stress echocardiograms in patients with high coronary risk profile
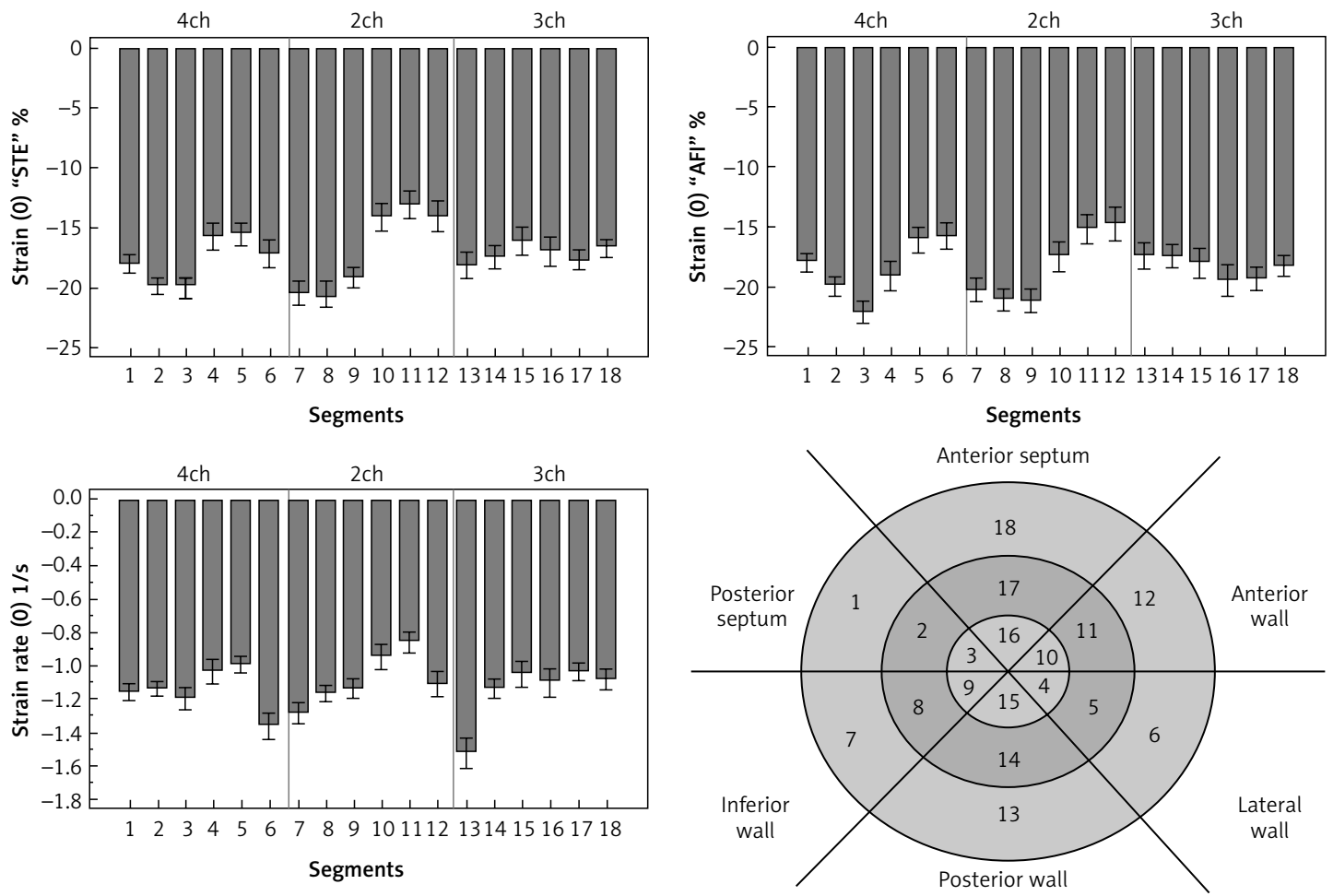

Figure 1. Mean values of regional systolic longitudinal strain and strain rate at baseline

STE - speckle tracking echocardiography, AFI - automated function imaging, 0 - baseline stage of dobutamine test, 4ch - four chamber segments visualized in four-chamber view, 2ch - two chamber segments visualized in two-chamber view, 3ch - three chamber segments visualized in three-chamber view. Numbers on bar graphs correspond to polar map location of individual segments.
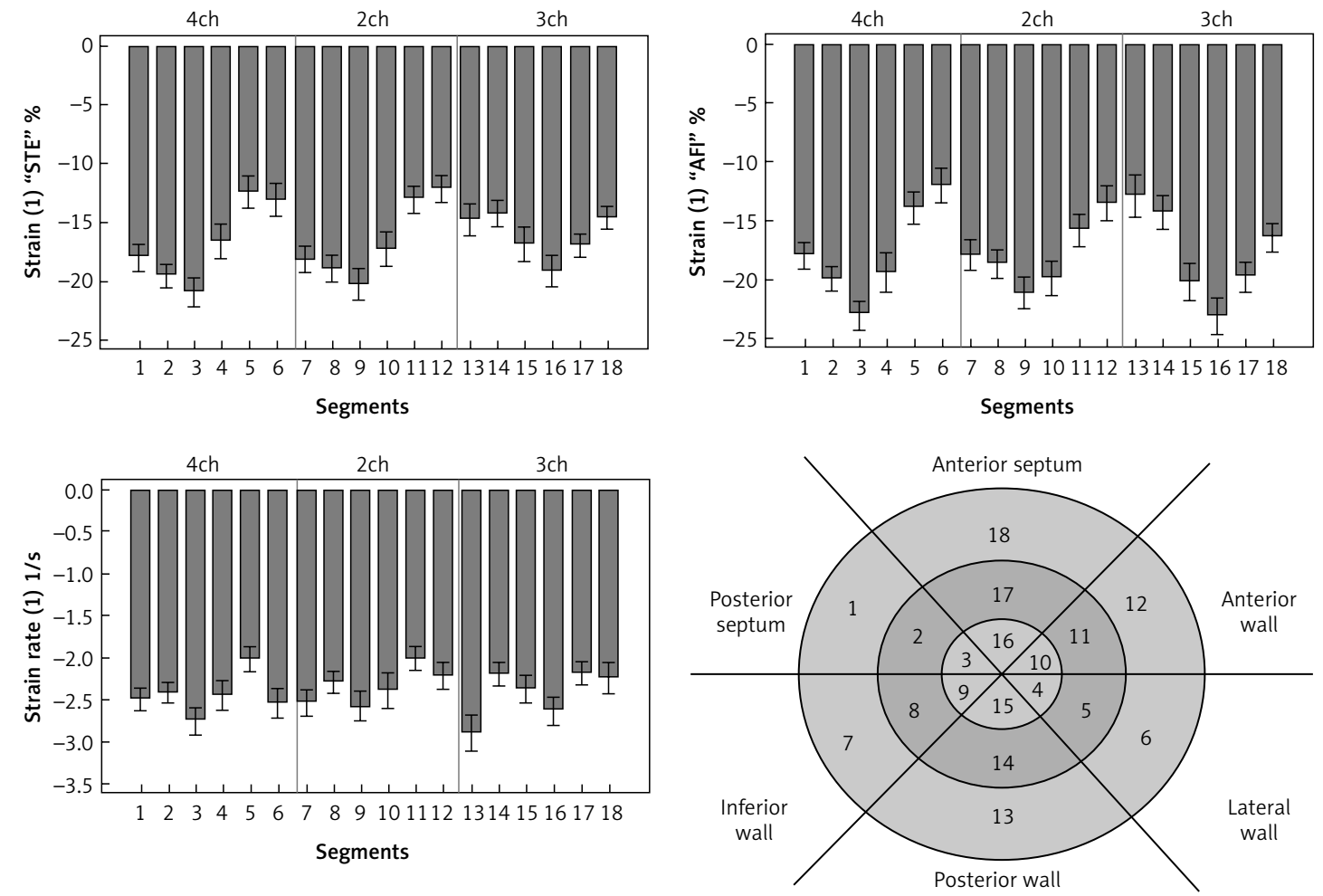

Figure 2. Mean values of regional systolic longitudinal strain and strain rate at peak stage of dobutamine stress echocardiography

STE - speckle tracking echocardiography, AFI - automated function imaging, 1-peak stage of dobutamine test, 4ch-four chamber segments visualized in four-chamber view, 2ch - two chamber segments visualized in two-chamber view, 3ch - three chamber segments visualized in three-chamber view. Numbers on bar graphs correspond to polar map location of individual segments. 

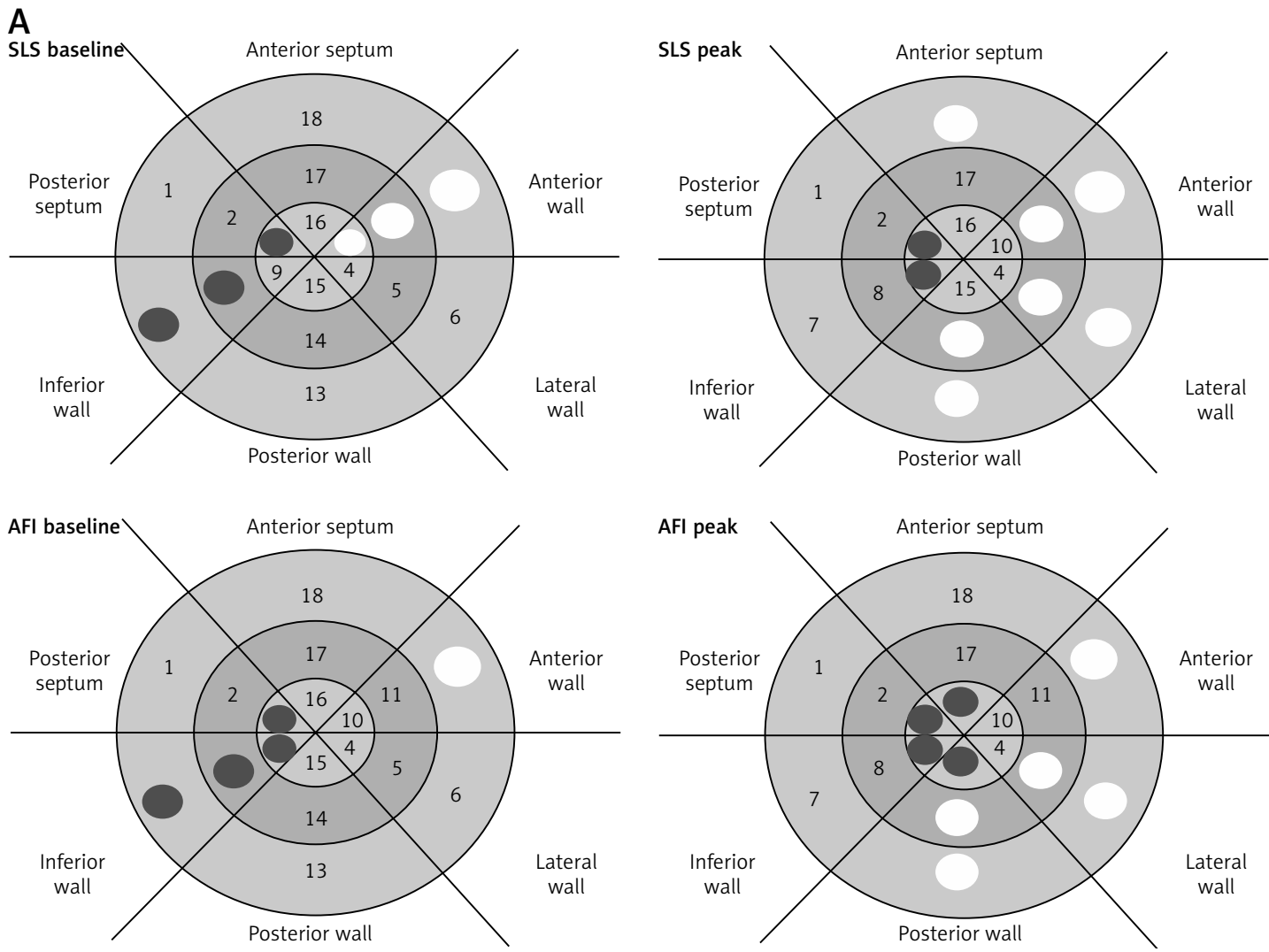

B
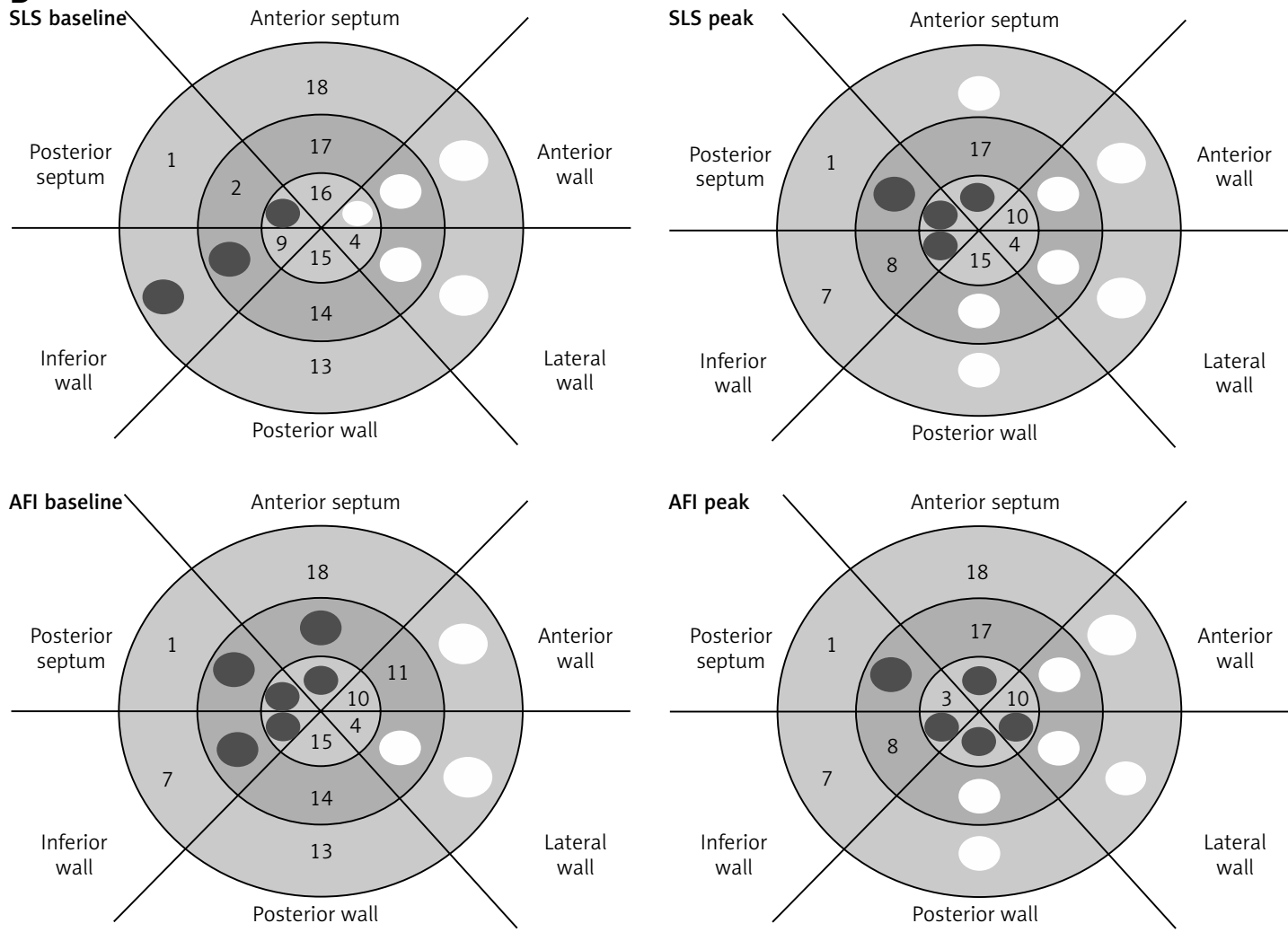

Figure 3. Segments with highest and lowest strain at baseline and peak stage of DSE. A - Polar plots showing segments with the highest $(>20 \%)$ and lowest $(<15 \%)$ absolute values of systolic longitudinal strain in patients without significant coronary stenoses. Dark speckles indicate segments with SLS $>20 \%$, white speckles indicate segments with SLS $<15 \%$. B - Polar plots showing segments with the highest (> 18\%) and the lowest (<14\%) absolute values of systolic longitudinal strain in patients with significant coronary stenoses. Dark speckles indicate segments with SLS > 18\%, white speckles indicate segments with SLS $<14 \%$ 


\section{Statistical analysis}

Statistical analysis was performed using MedCalc V. 12.1.4. (Frank Schoonjans Belgium). Continuous variables were expressed as means and standard deviations. Mean values of deformation parameters in 18 segments were compared with ANOVA or, when the equality of variances was not confirmed in Levene's test, with the Kruskal-Wallis test. $P<0.05$ was considered significant. The dispersion index for strain at baseline and at the peak stage of DSE was defined as the average of the segmental standard deviations of strain values in the segments composing specific regions. We calculated the dispersion index (DI) for all 18 LV segments and separately for basal, mid and apical regions. Comparison of DI at baseline and the peak stage of DSE was performed with the paired $t$-test. The coefficient of variation for duplicate measurements of regional SLS and AFI was calculated to assess intraobserver and interobserver variability for regional deformation in 15 randomly selected patients.

\section{Results}

The mean resting heart rate was $66 \pm 10$ beats per minute and increased during DSE to $143 \pm 12$, $p<0.001$. Similarly, a significant increase was observed for the systolic and diastolic blood pressure: from $127 \pm 17 \mathrm{~mm} \mathrm{Hg}$ at baseline to $143 \pm 27$ $\mathrm{mm} \mathrm{Hg}$ at stress $(p<0.001)$, and from $71 \pm 10 \mathrm{~mm} \mathrm{Hg}$ to $77 \pm 13 \mathrm{~mm} \mathrm{Hg}(p<0.001)$, respectively. The average achieved dose of dobutamine infusion was $35 \mu \mathrm{g} / \mathrm{kg} / \mathrm{min}$ and atropine dose $0.9 \pm 0.5 \mathrm{mg}$. The feasibility of deformation parameters was $\geq 95 \%$. Detailed data concerning the feasibility in consecutive LV segments were published previously [25].

Intraobserver and interobserver variability assessed for segmental values of strain in the basal segment of the posterior septum was $8.0 \%$ and $8.3 \%$ for SLS at baseline and $13.2 \%$ and $12.4 \%$ at peak. For AFI the respective coefficients of variance were $8.2 \%$ and $8.7 \%$ for baseline and $15.7 \%$ and $16.3 \%$ for peak stage measurements.

Significant heterogeneity of SLS values among the LV segments (measured both by AFI and STE, $p<0.001$ ) was observed at baseline and the peak stage of DSE. The lowest absolute values of SLS at baseline were observed in the anterior wall (segments 10, 11, 12, with SLS $14.2 \pm 6.2 \%, 13.1$ $\pm 6.2 \%$ and $14.1 \pm 6.9 \%$ respectively) and the highest in the basal and mid segment of the inferior wall (segments 7 and 8, with SLS $_{B} 20.5 \pm 5.2 \%$ and $20.8 \pm 4.6 \%)$ and in the apical segment of the posterior septum (segment 3 with $\mathrm{SLS}_{B} 20.1 \pm 4.6 \%$ ); see Figure 1. Similarly, resting strain values obtained by the AFI method were the lowest in the mid and basal anterior wall ( $\mathrm{AFI}_{\mathrm{B}} 15.2 \pm 6.6 \%$ and $14.8 \pm 7.5 \%$ in segments 11 and 12 respectively) and the highest in the apical segment of the posterior septum (segment 3, with $\mathrm{AFI}_{\mathrm{B}} 22.2 \pm 4.9 \%$ ) and in the mid and apical segments of the inferior wall (segments 8 and 9, with $\mathrm{AFI}_{\mathrm{B}} 21.2 \pm 4.6 \%$ and $21.3 \pm 5.3 \%$ ); see Table III.

During the peak stage of DSE, SLS was the lowest in the basal anterior (12.1 $\pm 5.9 \%)$ and mid lateral segments $(12.4 \pm 7.4 \%)$ and the highest in apical segments of the posterior septum (20.9 $\pm 6.4 \%)$ and inferior wall $(20.3 \pm 6.9 \%)$. In AFI analysis during the peak stage of DSE the lowest value was observed in basal segments of the lateral and posterior wall $(12.1 \pm 7.5 \%$ and $12.9 \pm 9.6 \%$ respectively) and the highest values in apical segments of the posterior and anterior septum (23 $\pm 7.1 \%$ and $23.2 \pm 8.7 \%$ ); see Figure 2 .

Since the results concerning regional analysis of all 18 segments are difficult to follow, we presented in schematic polar maps segments with the maximal ( $>20 \%$ ) and minimal absolute values $(<15 \%)$ of SLS and AFI during baseline and peak stages of DSE; see Figure 3. Additionally we analyzed the segmental SLS in 127 patients with significant stenoses of coronary arteries. The variability of strain values was still observed, with a similar pattern of segments with the highest and the lowest deformation as observed in the group without stenoses. Nevertheless, in patients with coronary artery disease (CAD) the absolute values of segmental strain were lower than in subjects without coronary lesions. We have presented a summary of our analysis of patients with CAD in panel B of Figure 3.

During DSE we observed an increase of the absolute value of SLS in apical LV segments (gray cells in Table III) and a decrease in basal and middle segments. These opposite directions of SLS changes were in the majority of segments confirmed in AFI analysis (Figure 4). Although systolic longitudinal strain rate (SLSR) values increased in all LV segments approximately twofold during dobutamine infusion, statistically significant differences between the segments emerged. The highest increase of SLSR was recorded in apical septal segments (posterior septum, $1.56 \pm 0.9 \mathrm{~s}^{-1}$ and anterior septum, $1.51 \pm 1.09 \mathrm{~s}^{-1}$ ) and the smallest increase in the middle segments of the lateral and posterior wall $\left(0.95 \pm 0.83 \mathrm{~s}^{-1}\right.$, and $\left.1.05 \pm 0.73 \mathrm{~s}^{-1}\right)$.

Dispersion indices (DI) of all deformation parameters (SLS, AFI and SLSR) calculated for the whole left ventricular muscle (18 segments) increased significantly during DSE $(p<0.0001$ for all). Baseline DI values for SLS, AFI and SLSR were respectively: $5.29 \pm 0.98,5.65 \pm 1.02$ and 0.33 \pm 0.07 . At the peak stage of DSE DI for SLS, AFI and SLSR increased respectively to: $6.53 \pm 0.89,7.44$ \pm 1.02 and $0.87 \pm 0.14$. A comparison of dispersion indices of SLS, AFI and SLSR between baseline and the peak stage of DSE is presented in Figure 5. The 
Table III. Comparison of the regional strain (classical and AFI) at baseline and at the peak stage of dobutamine stress echocardiography (DSE). The numbers in the headings of columns signify successive segments of the left ventricle as shown on polar maps in figures. Marked columns represent apical segments of the left ventricle

\begin{tabular}{|lccccccc|}
\hline Parameter & 1 & 2 & 3 & 4 & 5 & 6 & 7 \\
\hline $\mathrm{SLS}_{\mathrm{B}}(\%)$ & $-18 \pm 4$ & $-19.9 \pm 3.7$ & $-20.1 \pm 4.6$ & $-15.8 \pm 6$ & $-15.6 \pm 5.3$ & $-17.2 \pm 6$ & $-20.5 \pm 5.2$ \\
\hline $\mathrm{SLS}_{\mathrm{P}}(\%)$ & $-18 \pm 6.2$ & $-19.6 \pm 5.2$ & $-20.9 \pm 6.4$ & $-16.6 \pm 7.8$ & $-12.4 \pm 7.4$ & $-13.1 \pm 7.2$ & $-18.2 \pm 5.9$ \\
\hline$\Delta \mathrm{SLS}(\%)$ & $0.04 \pm 6.0$ & $0.33 \pm 5.2$ & $-0.82 \pm 6.5$ & $-0.73 \pm 7.4$ & $3.6 \pm 7.6$ & $4.5 \pm 8.5$ & $2.5 \pm 6.7$ \\
\hline $\mathrm{AFI}_{\mathrm{B}}(\%)$ & $-18 \pm 4.4$ & $-20 \pm 3.9$ & $-22.2 \pm 4.9$ & $-19.1 \pm 6.5$ & $-16.1 \pm 5.9$ & $-15.8 \pm 5.7$ & $-20.4 \pm 5.2$ \\
\hline $\mathrm{AFI}_{\mathrm{P}}(\%)$ & $-18 \pm 6.4$ & $-20 \pm 5.5$ & $-23 \pm 7.1$ & $-19.5 \pm 8.8$ & $-13.9 \pm 7$ & $-12.1 \pm 7.5$ & $-18 \pm 6.9$ \\
\hline$\Delta \mathrm{AFI}(\%)$ & $0.17 \pm 6.4$ & $0.05 \pm 5.5$ & $-0.79 \pm 7.1$ & $-0.38 \pm 8$ & $2.34 \pm 7.1$ & $3.93 \pm 8.65$ & $2.7 \pm 7.9$ \\
\hline
\end{tabular}

\begin{tabular}{|lcccccc|}
\hline Parameter & 8 & 9 & 10 & 11 & 12 & 13 \\
\hline $\mathrm{SLS}_{\mathrm{B}}(\%)$ & $-20.8 \pm 4.6$ & $-19.2 \pm 4.6$ & $-14.2 \pm 6.2$ & $-13.1 \pm 6.2$ & $-14.1 \pm 6.9$ & $-18.2 \pm 6$ \\
\hline $\mathrm{SLS}_{\mathrm{P}}(\%)$ & $-18.9 \pm 5.8$ & $-20.3 \pm 6.9$ & $-17.3 \pm 7.9$ & $-13.1 \pm 6.1$ & $-12.1 \pm 5.9$ & $-14.8 \pm 7.2$ \\
\hline$\Delta \mathrm{SLS}(\%)$ & $1.9 \pm 6.1$ & $-1.3 \pm 7.3$ & $-3.2 \pm 7.7$ & $0.2 \pm 6.8$ & $2.2 \pm 7.5$ & $3.5 \pm 8.3$ \\
\hline $\mathrm{AFI}_{\mathrm{B}}(\%)$ & $-21.1 \pm 4.6$ & $-21.3 \pm 5.3$ & $-17.5 \pm 7$ & $-15.2 \pm 6.6$ & $-14.8 \pm 7.5$ & $-17.5 \pm 5.8$ \\
\hline $\mathrm{AFI}_{\mathrm{P}}(\%)$ & $-18.7 \pm 6.5$ & $-21.2 \pm 6.8$ & $-19.9 \pm 8$ & $-15.8 \pm 7.4$ & $-13.6 \pm 7.6$ & $-12.9 \pm 9.6$ \\
\hline$\Delta \mathrm{AFI}(\%)$ & $2.4 \pm 6.9$ & $-0.06 \pm 6.8$ & $-2.4 \pm 7.3$ & $-0.33 \pm 7.1$ & $1.8 \pm 8.6$ & $5.0 \pm 9.6$ \\
\hline
\end{tabular}

\begin{tabular}{|lcccccc|}
\hline Parameter & 14 & 15 & 16 & 17 & 18 & $P$-value \\
\hline $\mathrm{SLS}_{\mathrm{B}}(\%)$ & $-17.5 \pm 5.1$ & $-16.2 \pm 6.2$ & $-17 \pm 6.4$ & $-17.8 \pm 4.2$ & $-16.7 \pm 4.0$ & $<0.001$ \\
\hline $\mathrm{SLS}_{\mathrm{P}}(\%)$ & $-14.3 \pm 6.1$ & $-16.9 \pm 7.8$ & $-19.2 \pm 7.1$ & $-17 \pm 5.4$ & $-14.6 \pm 5.3$ & $<0.001$ \\
\hline$\Delta \mathrm{SLS}(\%)$ & $3.3 \pm 6.4$ & $-0.72 \pm 8.5$ & $-2.0 \pm 9.3$ & $0.75 \pm 6.8$ & $2.2 \pm 6.7$ & $<0.001$ \\
\hline $\mathrm{AFI}_{\mathrm{B}}(\%)$ & $-17.5 \pm 5.3$ & $-18 \pm 6.7$ & $-19.5 \pm 6.8$ & $-19.4 \pm 5.1$ & $-18.3 \pm 4.5$ & $<0.001$ \\
\hline $\mathrm{AFI}_{\mathrm{P}}(\%)$ & $-14.3 \pm 7.7$ & $-20.3 \pm 8.7$ & $-23.2 \pm 8.7$ & $-19.9 \pm 7$ & $-16.5 \pm 6.7$ & $<0.001$ \\
\hline$\Delta \mathrm{AFI}(\%)$ & $3.3 \pm 7.5$ & $-2.1 \pm 9.2$ & $-3.9 \pm 9.3$ & $-0.5 \pm 7.5$ & $1.7 \pm 7.5$ & $<0.001$ \\
\hline
\end{tabular}

SLS - systolic longitudinal strain, B - baseline stage of DSE, $P$ - peak stage of DSE, $\Delta$ - change between baseline and peak stage.

calculation of a separate dispersion index for basal, middle and apical segments of the LV revealed the highest values of DI in apical LV segments and the lowest in mid segments, although these differences achieved significance only at the peak stage of DSE (Table IV).

Our results indicate significant variability of regional deformation parameters of LV at rest and at the peak stage of DSE in the subjects without significant coronary artery stenoses. The resting SLS presented the lowest values in the anterior and lateral wall, supplied usually by the left coronary artery, and the highest values in the inferior wall and posterior septum, which belong to the territory of the right coronary artery. At the peak stage of dobutamine infusion, the highest amplitudes of strain were registered in the apical segments, although the pattern of higher strain in the right coronary artery region was generally preserved; for an example of DSE in a woman with normal coronary arteries (Figure 6).
In our study we did not observe fatal or life-threatening complications related directly to DSE in the whole studied group of 250 patients. The analysis of DSE in 111 patients without significant coronary stenoses revealed 14 adverse events including: 2 cases of prolonged stenocardial pain, 2 hypertensive reactions, numerous ventricular extrasystoles in 5 patients, and single events of hypotonia, bradycardia, induction of left bundle branch block, spontaneously remitting supraventricular tachycardia and atrial fibrillation managed by electrical cardioversion on the next day. The percentage of these mild complications was $12.6 \%$, which is close to the value of $10 \%$ of complications during stress tests reported in the literature.

\section{Discussion}

According to our knowledge, only a handful of studies evaluating the variability of regional deformation parameters have been published to 

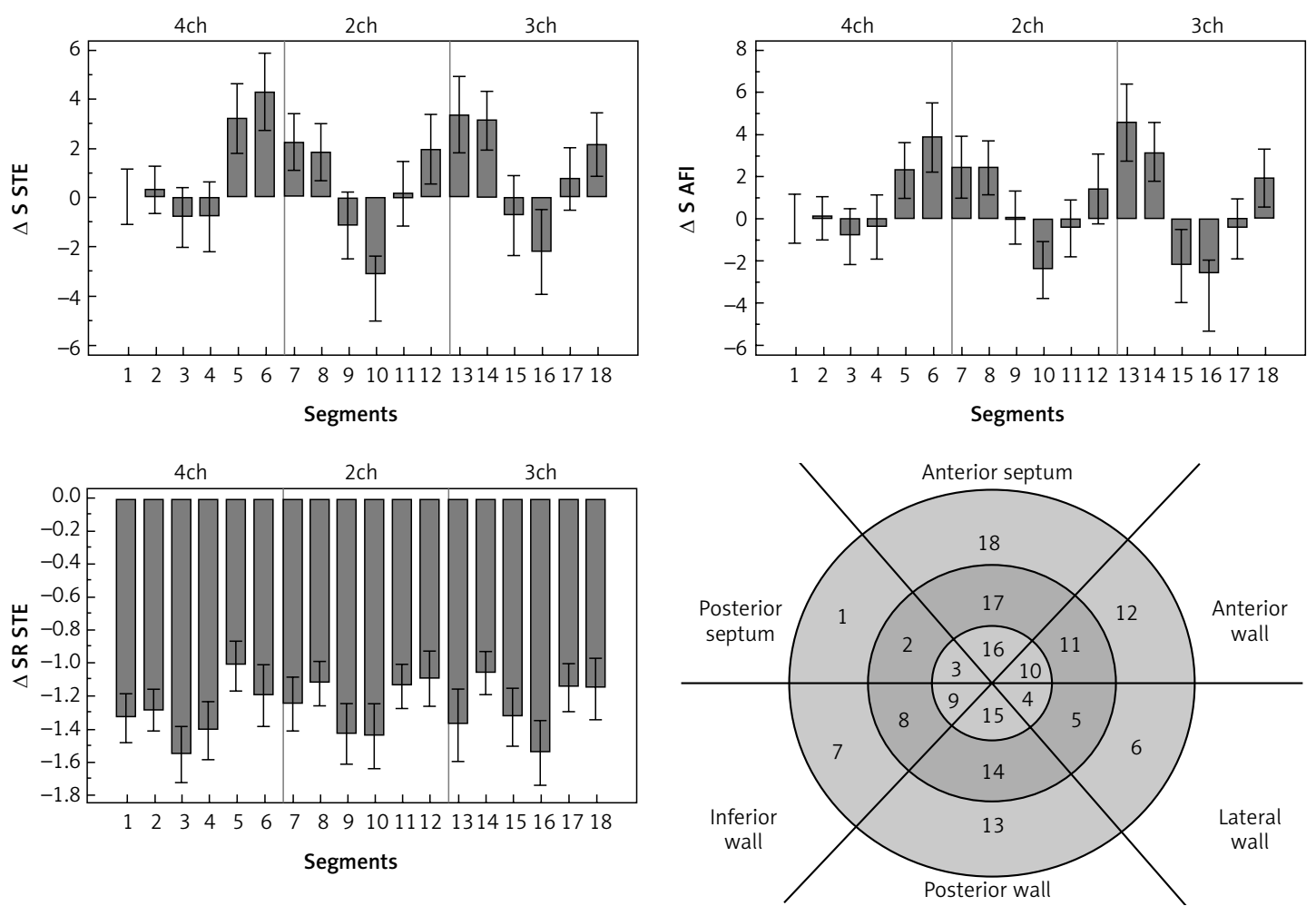

Figure 4. Mean values of changes in regional systolic longitudinal strain and strain rate between peak and baseline stage of dobutamine stress echocardiography

STE - speckle tracking echocardiography, AFI-automated function imaging, S-strain, SR - strain rate, $\Delta$-change between peak and baseline stage of dobutamine test for respective parameter, 4ch - four chamber segments visualized in four-chamber view, $2 \mathrm{ch}$ - two chamber segments visualized in two-chamber view, 3ch - three chamber segments visualized in three-chamber view. Numbers on bar graphs correspond to polar map location of individual segments.

date, and papers concerning heterogeneity during DSE or other stress tests are still unique.

Afonso et al. [26] postulated that the strain dispersion index reflecting non-uniformity of myocardial function may be helpful in differentiation between hypertrophic cardiomyopathy and hypertrophy in athletes and hypertensives. Subjects with hypertrophic cardiomyopathy presented not only lower absolute values of global longitudinal strain but also higher dispersion of regional strain $(\mathrm{DI}=4.6 \pm 1.7)$ in comparison with hypertensive $(\mathrm{DI}=3.5 \pm 1)$ and athletic hypertrophy $(\mathrm{DI}=2.6$ \pm 0.5 ). Hypertensive hypertrophy was characterized by similar global strain values to those in athletes (which in turn were close to values observed in healthy subjects) but differed in the significantly higher dispersion index of strain. Calculated in an analogous way, in our study the dispersion index of strain measured by the AFI method was 5.65 \pm 1.02 at rest, which was more similar to values observed in subjects with myocardial hypertrophy than the significantly younger and very small control group in Afonso's study (the mean age of 12 subjects of the control group was $29.3 \pm 6.3$ years). However, mean values of systolic and early diastolic mitral annulus motion measured at rest in our group corresponded to well-preserved systolic and diastolic function (see Table II).
Regarding the profile of regional strain in healthy subjects, Sun et al. [27] observed higher value of longitudinal and circumferential strain in apical versus basal segments. In the study of Marwick et al. [28], higher values of longitudinal strain at rest were observed in the majority of apical segments and, similarly to our results, in the inferior wall. Also the more recent paper dedicated to determination of reference values of strain

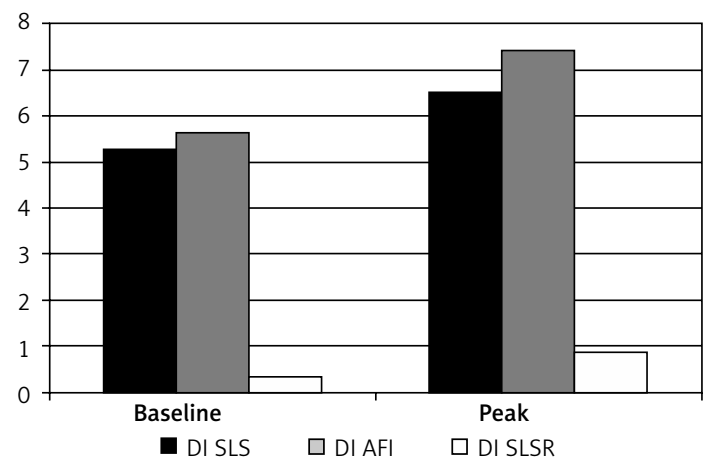

Figure 5. Comparison of dispersion indices (DI) of deformation parameters between baseline and peak stage of DSE. Black bars indicate DI of SLS, gray bars indicate DI of AFI, and white bars indicate DI of SLSR

$p<0.0001$ for all comparisons between baseline and peak 
Table IV. Comparison of dispersion indexes between basal, middle and apical segments of the left ventricle

\begin{tabular}{|lcccc|}
\hline Parameter & Basal segments & Mid segments & Apical segments & $P$-value \\
\hline$S L S_{B}$ & $5.35 \pm 1.18$ & $4.85 \pm 0.88$ & $5.67 \pm 0.84$ & NS \\
\hline$S L S_{P}$ & $6.28 \pm 0.77$ & $6.00 \pm 0.78$ & $7.32 \pm 0.61$ & 0.016 \\
\hline$A F I_{B}$ & $5.52 \pm 1.13$ & $5.23 \pm 0.95$ & $6.20 \pm 0.88$ & $\mathrm{NS}$ \\
\hline AFI $_{P}$ & $7.78 \pm 1.18$ & $6.85 \pm 0.78$ & $8.02 \pm 0.88$ & $\mathrm{NS}$ \\
\hline $\mathrm{SLSR}_{\mathrm{B}}$ & $0.35 \pm 0.08$ & $0.28 \pm 0.04$ & $0.37 \pm 0.05$ & $\mathrm{NS}$ \\
\hline $\mathrm{SLSR}_{\mathrm{P}}$ & $0.93 \pm 0.15$ & $0.73 \pm 0.05$ & $0.95 \pm 0.08$ & 0.004 \\
\hline
\end{tabular}

$S L S$ - systolic longitudinal strain, AFI - systolic longitudinal strain measured by automated function imaging, SLSR - systolic longitudinal strain rate, $B$ - baseline stage of DSE, $P$ - peak stage of DSE.
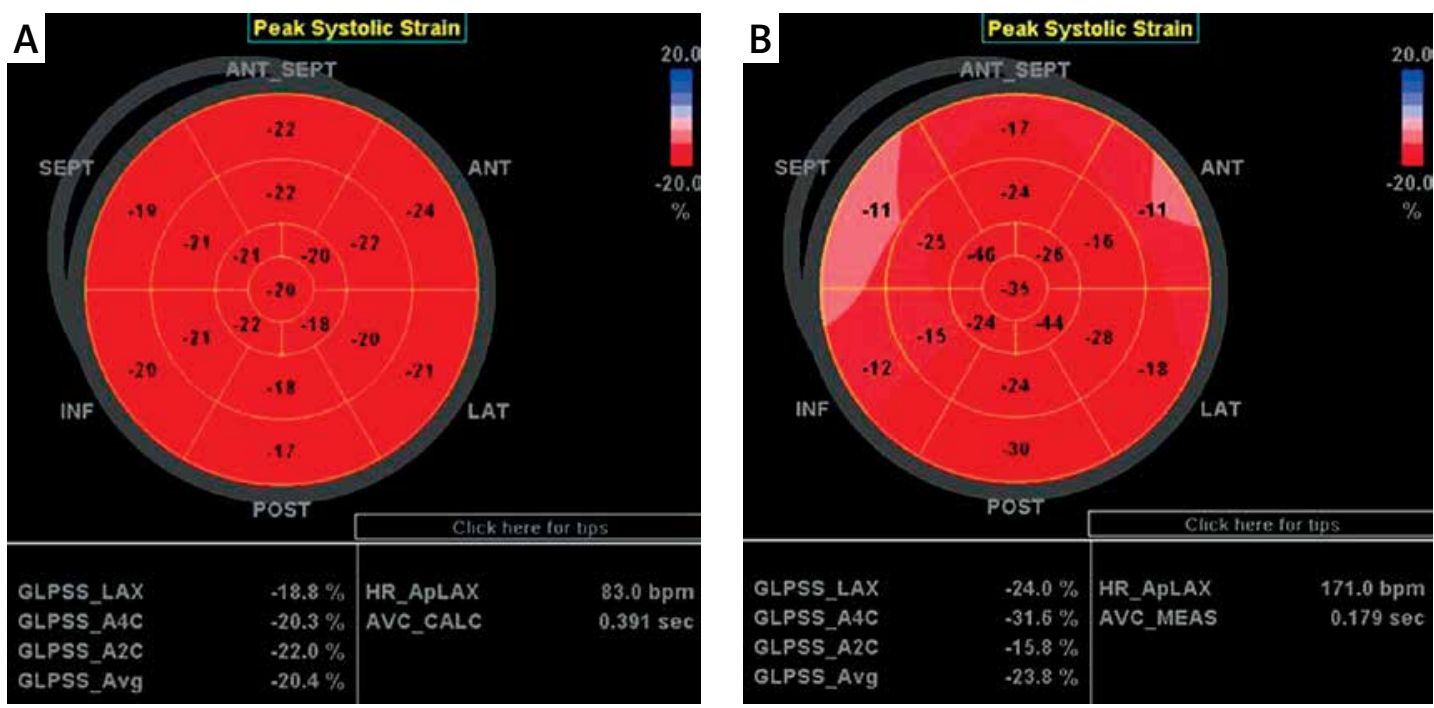

Figure 6. Strain patterns during baseline and peak stage of DSE in a woman without coronary artery disease. Panel A presents polar plot obtained at baseline at heart rate 83 beats/minute. Range of absolute values of segmental strain is from $17 \%$ to $24 \%$, mean absolute value of segmental strain in apical region is $20 \%$, in mid-segments $21 \%$, and in basal segments $21 \%$. Panel B presents polar plot obtained at peak DSE at heart rate 171 beats/minute. Range of absolute values of segmental strain is from $11 \%$ to $46 \%$, mean absolute value of segmental strain in apical region is $35 \%$, in mid-segments $22 \%$, and in basal segments $17 \%$. A distinct increase was observed according to the absolute value of regional strain in the apical region, whereas in the basal region a mild decrease was observed

by three-dimensional speckle tracking echocardiography revealed significant heterogeneity in longitudinal strain between individual segments [29]. It seems possible that the observed variability of deformation reflects not only the complex geometry and three-layer architecture of the LV but also some differences of perfusion in the field of both coronaries. In the left anterior descending artery the dominance of the diastolic phase of the flow spectrum is more evident than in the right coronary artery, which may have some impact on contractile function, especially during tachycardia at the peak stage of DSE.

Interestingly, also SLS changes observed during DSE displayed significant region-related dispersion, with an increase in the apical and decrease in the basal and middle LV segments. This opposite direction of changes in regional deformation at different LV levels indicates the need for differentiation of segment- or region-specific cut-off values for deformation-based ischemia detection during DSE.

We observed a significant increase of dispersion for strain and strain rate at the peak stage of DSE, and at this stage of the stress test segmental non-uniformity was more pronounced in the apical and less so in mid LV segments (Figure 5 and Table IV). A potential solution of this problem is the choice of marker segments, representative for the territory of each coronary artery, from mid $\mathrm{LV}$ segments presenting with lower dispersion indices.

Poorly examined issues also include the changes and dispersion of regional strain during other stress tests such as exercise on a treadmill, pacing or dipyridamole studies. Nowadays, the changes of deformation during exercise are intensively studied and sometimes reveal data which (analyzed in abstraction from achieved heart rate) seem to be contradictory, indicating e.g. physio- 
logic lack of increase of longitudinal strain during maximal exercise (swimming) in healthy athletes [30] and poor prognosis related to lowered global longitudinal strain during moderate exercise (bicycle, level of HR 90-100 beats per minute for speckle tracking analysis) in patients with heart failure and preserved ejection fraction [31]. Despite the lack of data concerning variability of segmental deformation, it seems probable that the main determinant of strain values lies primarily in heart rate and inotropic excitement achieved rather than in specificity of the applied stressor.

Our study has several limitations. Our group was limited in size and the subjects did not undergo a study of deformation with an alternative method, e.g. myocardial tagging in magnetic resonance imaging. We studied patients without significant lesions of coronary arteries but with a high percentage of cardiovascular risk factors and chest pain. Therefore our results do not necessarily reflect the findings in normal subjects but still may serve as a reference for typical patients referred for a stress test. The assessment of coronary arteries was based on diameter assessed by an experienced invasive cardiologist but was not supported by advanced approaches, e.g. fractional flow reserve or intracoronary ultrasound.

The significant burden of CAD risk factors, such as hypertension and diabetes, and cardiovascular medications may have influenced myocardial function although probably in a homogeneous manner without a predilection to specific regions [32]. We focused on the comparisons of dispersion indices between baseline and the peak stage of DSE and in separate regions of the LV, did not assess the impact of age, sex or regional thickness of the myocardium on variability of strain, and did not analyze the potential relationship of strain heterogeneity with different types of coronary circulation: right or left coronary artery dominance. Finally, our analysis was limited to the amplitudes of deformation parameters, whereas variability of time intervals may also provide significant information [33].

In conclusion, our data indicate heterogeneity of segmental deformation in patients without significant lesions in coronary arteries undergoing stress testing. This variability is related to basal, middle or apical localization of LV segments and the potential relationship with supplying coronary artery. The dispersion is the most evident in apical segments and increases during the peak stage of DSE. The DSE-induced changes of longitudinal strain, but not strain rate, are opposite in direction in the basal/middle vs. apical part of the LV. Further evaluation is required to determine whether these observations have an impact in the setting of coronary artery disease.

\section{Acknowledgments}

The work was supported by a grant of the State Committee for Scientific Research, number N N402 500240.

\section{Conflict of interest}

The authors declare no conflict of interest.

\section{References}

1. Sicari R, Nihoyannopoulos P, Evangelista A, et al. EAE Guidelines Stress echocardiography expert consensus statement European Association of Echocardiography (EAE) (a registered branch of the ESC). Eur J Echocardiogr 2008; 9: 415-37.

2. Elhendy A, van Domburg RT, Bax JJ, et al. Optimal criteria for the diagnosis of coronary artery disease by dobutamine stress echocardiography. Am J Cardiol 1998; 82: 1339-44.

3. Kukulski T, Jamal F, Herbots L, et al. Identification of acutely ischemic myocardium using ultrasonic strain measurements. A clinical study in patients undergoing coronary angioplasty. J Am Coll Cardiol 2003; 41: 810-9.

4. Langeland S, D'Hooge J, Wouters P, et al. Experimental validation of a new ultrasound method for the simultaneous assessment of radial and longitudinal myocardial deformation independent of insonation angle. Circulation 2005; 112: 2157-62.

5. Geyer H, Carraciolo G, Abe H, et al. Assessment of myocardial mechanics using speckle tracking echocardiography: fundamentals and clinical applications. J Am Soc Echocardiogr 2010; 23: 351-69.

6. Drożdż J, Krzemińska-Pakuła M, Plewka M, Ciesielczyk M, Kasprzak JD. Prognostic value of low-dose dobutamine echocardiography in patients with idiopathic dilated cardiomyopathy. Chest 2002; 121: 1216-22.

7. Wierzbowska-Drabik K, Roszczyk N, Sobczak M, et al. Effect of sex on diagnostic efficacy of dobutamine stress echocardiography with early atropine administration in the detection of coronary artery disease. Pol Arch Med Wewn 2014; 124: 105-13.

8. Yurdakul S, Yildirimturk O, Aytekin S. Left atrial mechanical functions in chronic primary mitral regurgitation patients: a velocity vector imaging-based study. Arch Med Sci 2014; 10: 455-63.

9. Płońska-Gościniak E, Lipiec P, Lancellotti P, et al. Prognostic value of low-dose dobutamine stress echocardiography in patients with aortic stenosis and impaired left ventricular function. Arch Med Sci 2013; 9: 434-9.

10. Vukajlovic D, Milasinovic G, Angelkov L, et al. Contractile reserve assessed by dobutamine test identifies super-responders to cardiac resynchronization therapy. Arch Med Sci 2014; 10: 684-91.

11. Katz WE, Gulati VK, Mahler CM, Gorcsan J $3^{\text {rd }}$. Quantitative evaluation of the segmental left ventricular response to dobutamine stress by tissue Doppler echocardiography. Am J Cardiol 1997; 79: 1036-42.

12. Cain P, Baglin T, Case C, Spicer D, Short L, Marwick TH. Application of tissue Doppler to interpretation of dobutamine echocardiography and comparison with quantitative coronary angiography. Am J Cardiol 2001; 87: 525-31.

13. Madler CF, Payne N, Wilkenshoff U, et al. Non-invasive diagnosis of coronary artery disease by quantitative stress echocardiography: optimal diagnostic models 
using off-line tissue Doppler in the MYDISE study. Eur Heart J 2003; 24: 1584-94.

14. Heimdal A, Støylen A, Torp H, Skjærpe T. Real-time strain rate imaging of the left ventricle by ultrasound. J Am Soc Echocardiogr 1998; 11: 1013-9.

15. Urheim S, Edvardsen T, Trop, Angelsen B, Smiseth OA. Myocardial strain by Doppler echocardiography: validation of a new method to quantify regional myocardial function. Circulation 2000; 102: 1158-64.

16. Weidemann F, Kowalski M, D'Hoge, Bijnens B, Sutherland GR. Doppler myocardial imaging. A new tool to assess regional inhomogeneity in cardiac function. Basic Res Cardiol 2001; 96: 595-605.

17. Zamojska J, Niewiadomska-Jarosik K, Wosiak A, Stańczyk J. Evaluation of left ventricular systolic function with the use of tissue Doppler echocardiography in children with primary arterial hypertension. Pol J Cardiol 2012; 14: 95-100.

18. D'Hooge J, Heimdal A, Jamal F, et al. Regional strain and strain rate measurements by cardiac ultrasound: principles, implementation and limitations. Eur J Echocardiogr 2000; 1: 154-70.

19. Chrzanowski Ł, Lipiec P, Krzemińska-Pakuła M, et al. Comparison of apical left ventricular segments strain imaging by tissue Doppler and speckle tracking echocardiography. Kardiol Pol 2008; 66: 388-93.

20. Hanekom L, Cho GY, Leano R, Jeffriess L, Marwick TH Comparison of two-dimensional speckle and tissue Doppler strain measurement during dobutamine stress echocardiography: an angiographic correlation. Eur Heart J 2007; 28: 1765-72.

21. Belghiti $H$, Brette $S$, Lafitte $S$, et al. Automated function imaging: a new operator-independent strain method for assessing left ventricular function. Arch Cardiovasc Dis 2008; 101: 163-9.

22. Sachdev A, Villarraga HR, Frantz RP, et al. Right ventricular strain for prediction of survival in patients with pulmonary arterial hypertension. Chest 2011; 139: 1299-309.

23. Kim HS, Cho Kl. Impact of chronic emotional stress on myocardial function in postmenopausal women and its relationships with endothelial dysfunction. Korean Circ J 2013; 43: 295-302.

24. Dibble CT, Lima JAC, Bluemke DA. et al. Regional left ventricular systolic function and the right ventricle. The multi-ethnic study of atherosclerosis right ventricle study. Chest 2011; 140: 310-6.

25. Wierzbowska-Drabik K, Hamala P, Roszczyk N, et al. Feasibility and correlation of standard 2D speckle tracking echocardiography and automated function imaging derived parameters of left ventricular function during dobutamine stress test. Int J Cardiovasc Imaging 2014; 30: 729-37.

26. Afonso L, Kondur A, Simegn M, et al. Two-dimensional strain profiles in patients with physiological and pathological hypertrophy and preserved left ventricular systolic function: a comparative analyses. BMJ Open 2012; 2(4). doi: 10.1136/bmjopen-2012-001390. Print 2012.

27. Sun JP, Lee AP, Wu C, et al. Quantification of left ventricular regional myocardial function using two-dimensional speckle tracking echocardiography in healthy volunteers- a multi-center study. Int J Cardiol 2013; 167: 495-501.

28. Marwick T, Leano RL, Brown J, et al. Myocardial strain measurement with 2-dimensional speckle tracking echocardiography. Definition of normal range. J Am Coll Cardiol Img 2009; 2: 80-4.

29. Kleijn S, Pandian NG, Thomas JD, et al. Normal reference values of left ventricular strain using three-dimensional speckle tracking echocardiography: results from a multicentre study. Eur Heart J Cardiovasc Imaging 2015; 16 410-6.

30. Santoro A, Alvino F, Antonelli G, et al. Left ventricular strain modifications after maximal exercise in athletes: a speckle tracking study. Echocardiography 2015; 32: 920-7.

31. Wang J, Fang F, Wai-Kwok Yip G, et al. Left ventricular long-axis performance during exercise is an important prognosticator in patients with heart failure and preserved ejection fraction. Int I Cardiol 2014; 23: 131-5.

32. Możdżan M, Wierzbowska-Drabik K, Kurpesa M, et al. Echocardiographic indices of left ventricular hypertrophy and diastolic function in hypertensive patients with preserved LVEF classified as dippers and non-dippers. Arch Med Sci 2013; 9: 268-75.

33. Santos AB, Kraigher-Krainer E, Bello N, et al. Left ventricular dyssynchrony in patients with heart failure and preserved ejection fraction. Eur Heart J 2014; 35: 42-7. 